\title{
TASC-MADM: Task Assignment in Spatial Crowdsourcing Based on Multiattribute Decision-Making
}

\author{
Yunhui Li $\mathbb{D},^{1}$ Liang Chang $\mathbb{D}^{2},{ }^{2}$ Long Li $\mathbb{D}^{2},{ }^{2}$ Xuguang Bao $\mathbb{D}^{2},{ }^{2}$ and Tianlong Gu $\mathbb{D}^{3}$ \\ ${ }^{1}$ School of Information and Communication, Guilin University of Electronic Technology, Guilin 541004, China \\ ${ }^{2}$ Guangxi Key Laboratory of Trusted Software, Guilin University of Electronic Technology, Guilin 541004, China \\ ${ }^{3}$ College of Information Science and Technology, Jinan University, Guangzhou 510000, China
}

Correspondence should be addressed to Tianlong Gu; gutianlong@jnu.edu.cn

Received 7 May 2021; Accepted 9 August 2021; Published 21 August 2021

Academic Editor: Qing Yang

Copyright ( $\odot 2021$ Yunhui Li et al. This is an open access article distributed under the Creative Commons Attribution License, which permits unrestricted use, distribution, and reproduction in any medium, provided the original work is properly cited.

\begin{abstract}
The methodology, formulating a reasonable task assignment to find the most suitable workers for a task and achieving the desired objectives, is the most fundamental challenge in spatial crowdsourcing. Many task assignment approaches have been proposed to improve the quality of crowdsourcing results and the number of task assignment and to limit the budget and the travel cost. However, these approaches have two shortcomings: (1) these approaches are commonly based on the attributes influencing the result of task assignment. However, different tasks may have different preferences for individual attributes; (2) the performance and efficiency of these approaches are expected to be improved further. To address the above issues, we proposed a task assignment approach in spatial crowdsourcing based on multiattribute decision-making (TASC-MADM), with the dual objectives of improving the performance as well as the efficiency. Specifically, the proposed approach jointly considers the attributes on the quality of the worker and the distance between the worker and the task, as well as the influence differences caused by the task's attribute preference. Furthermore, it can be extended flexibly to scenarios with more attributes. We tested the proposed approach in a realworld dataset and a synthetic dataset. The proposed TASC-MADM approach was compared with the RB-TPSC and the BudgetTASC algorithm using the real dataset and the synthetic dataset; the TASC-MADM approach yields better performance than the other two algorithms in the task assignment rate and the CPU cost.
\end{abstract}

\section{Introduction}

Spatial crowdsourcing, first introduced by Kazemi and Shahabiin [1], refers to an economic and efficient solution to participation in completing tasks, such as sensing tasks $[2,3]$. The popularity of mobile devices and advanced Internet technologies have made it a popular trend in performing spatial tasks $[4,5]$. Unlike conventional crowdsourcing, spatial crowdsourcing requires a worker to travel to a given location to perform a given task [6]. Examples of spatial crowdsourcing, such as environmental conditions and monitoring traffic flow at selected locations $[7,8]$, crowdsourcing news reporting tasks [9], and natural disaster response [10], are likely to have spatial requirements that cannot be fulfilled remotely and require physical arrival at the task's location. Spatial crowdsourcing is becoming a compelling paradigm for recruiting workers to perform the tasks. However, due to the openness of crowdsourcing, there are some core issues: (1) how to guarantee the quality of crowdsourcing results and the number of tasks completed; (2) how to control the cost, such as the budget used and the travel cost; and (3) how to ensure the efficiency of task completed. All three core issues in spatial crowdsourcing are involved in task assignment. Thus, the task assignment is considered as the most fundamental challenge in spatial crowdsourcing [11].

The task assignment approach is mainly based on some attributes affecting the performance of task assignments [12], such as the distances between workers and tasks, and the qualities of workers. On one hand, previous research has shown that a task's distance from a worker affects the crowdsourcing outcomes $[13,14]$. Tasks that are further 
away from workers are less likely to be completed because workers tend to complete the nearby tasks. Some task assignment algorithms consider the locations of the tasks and workers to maximize the total number of assigned tasks $[1,14,15]$. On the other hand, the quality of a worker is considered to positively affect the crowdsourcing result's quality [16]. Some works evaluate a worker's quality by using distance and reputation and then propose a task allocation approach that balances the result's quality and the budget utilization rate $[16,17]$. The existing task assignment method is based on the attributes that influence the results but ignores the different preferences of a task for different attributes. For example, monitoring traffic flow has strict requirements on the location of a worker, monitoring climate may accept workers working in a slightly larger area such as a city, and reporting news has limitations on both the location and quality of workers. Therefore, different tasks may have different attribute preferences.

Studies on spatial task assignment aim to allocate the task to suitable workers for different objectives, such as maximizing the total amount of assigned tasks $[1,18]$, minimizing the total travel cost of the allocated workers [16], and maximizing the overall quality of crowdsourcing results under the budget constraint [19]. Unfortunately, these objectives conflict with each other; optimizing multiple goals simultaneously is especially difficult. Consider the following examples: (a) increasing the total amount of tasks assigned is potentially achieved by relaxing the constraints on workers, such as increasing the accepted task region in which the task is allowed to be performed and lowering the threshold of workers' credibility. However, these methods may lead to increased travel costs or decreased quality of results. (b) One way to reduce the uncertainty of crowdsourced data is to ask multiple workers to complete the same task and then aggregate the responses of those workers to get the result of the task. However, asking multiple workers to complete the same task will increase the payment and the latency. Thus, a task assignment solution must involve a trade-off among various objectives.

In short, (1) the existing task assignment approach does not fully consider the task's attribute preference; (2) a specific task assignment approach usually achieves a certain objective, but fails to achieve several conflicting goals. To address the two problems, we propose a flexible and efficient task assignment approach in spatial crowdsourcing based on multiattribute decision-making (TASC-MADM), which takes into account the distance attribute and the reputation attribute simultaneously, as well as tasks' different preferences of attributes. Our goal is to trade off the quality of the result and the task allocation rate under the budget constraint of the task.

In this paper, we advance the key contributions of our research as follows:

(i) Unlike existing work that simply focuses on some critical attributes while ignoring the preferences of different tasks, in this paper, we collectively consider the impact of distance attribute and worker quality attribute on the crowdsourcing result, as well as tasks' different preferences for attributes. (ii) We formulate the problem of task assignment in spatial crowdsourcing as a multiattribute decisionmaking (TASC-MADM) problem and propose a novel algorithm solving this problem. The linear weighted-evaluation method is used to rating the candidate workers comprehensively, which enables a task to select the appropriate worker according to its preference for attributes. Besides, the proposed approach allows achieving the objectives of maximizing the total amount of assigned tasks or the quality of outcome by setting attribute weights.

(iii) Although our algorithm is simple, it performs well. Besides, it can be flexibly extended to the situation where any number of attributes affects the crowdsourcing result.

The rest of the paper is organized as follows. Section 2 presents related works on task assignment of spatial crowdsourcing. Section 3 formally defines problems involved in TASC-MADM. Section 4 describes the proposed TASC-MADM approach. The performance evaluation and discussion of the TASC-MADM approach are conducted in Section 5. Finally, Section 6 concludes the work and suggests some directions for future studies.

\section{Related Work}

Task assignment, i.e., the intelligent matching of tasks with the most appropriate workers, is a fundamental challenge of crowdsourcing [20-22]. Although there have been several studies on conventional crowdsourcing task allocation, they cannot be directly applied to spatial crowdsourcing, because the location of the spatial task and that of the workers are vital for the result of the spatial task assignment.

Research on spatial crowdsourcing task allocation is still in the early stage. In the spatial task assignment area, existing studies have mainly concentrated on exploiting the attributes of the tasks and workers. These attributes usually indicate the distance between the location of the workers and tasks, the capacity (i.e., the maximum number of tasks that a worker is willing/able to complete), and the quality of the workers [21, 23], etc. Kazemi and Shahabi [1] utilized the spatial region $R$ (i.e., a rectangle region in which the worker accepts tasks) and the capacity of the workers max $T$ to assign each worker to his nearby tasks. The greedy (GR) algorithm is presented to maximize the task assignment at each time instance. However, the greedy strategy cannot solve the global optimization problem. To solve this problem, heuristics are used to maximize the overall assignments. Hence, they proposed the second strategy: the least location entropy priority (LLEP) strategy. A location located in an area with few workers has low entropy. Conversely, a location located in a worker-density area has high entropy. Obviously, tasks with smaller location entropy are less likely to be completed by workers. In the heuristic, a higher priority is given to tasks located in areas with smaller location entropy. Furthermore, travel cost is a critical issue for spatial crowdsourcing. High travel costs may prevent workers from participating in the task and result in high costs for task requesters. Hence, they 
proposed the third strategy: the nearest neighbor priority (NNP) strategy. Workers are assigned to tasks closer to them in preference, which aims at maximizing the overall finished tasks while reducing the workers' travel cost whenever possible. The research of [1] aims to maximize the number of task assignments while keeping the travel cost minimized, but they assume that the worker does not reject tasks assigned to them and trusts the workers to be reliable. Hassan and Curry [24] consider the situation where the worker can reject a task, propose a contextual bandit algorithm learning the possibility of task accepted by a worker, to assign a worker with high possibility based on the spatial locations of workers and tasks, and aim to maximize the total amount of successful assignments.

In addition to the locations of the tasks and workers and the capacity of workers, the quality of workers is another important attribute affecting the result of task allocation. Some works incorporate the quality of workers in the assignment process with the aim of controlling the quality [25] and cost for all completed tasks $[16,19,26]$. In traditional crowdsourcing, worker quality can be modeled by the worker's reputation [26-28], which may be a rating of the worker's past works, or an evaluation of the worker's knowledge, ability, confidence in completing tasks successfully, etc. A worker with a higher reputation is generally perceived to be better at his work. Cheng et al. [29] considered workers' confidence in completing tasks successfully and proposed the reliable-diversity-based spatial crowdsourcing approach. The approach Budget-TASC [16] considers the number of workers in the task assignment and thinks that the distance of a worker from the task negatively influences the quality of the crowdsourcing result $[17,18]$; the reliability of the workers is given by a reputation function discounted by the distance. Then, the task is assigned to the worker with the highest reliability, to maximize the desired quality of results obtained, while the total budget is limited. However, the task assignment rate of spatial crowdsourcing tasks is not considered. RB-TPSC [17] presents a task package assignment algorithm with aim of maximizing the desired quality of the results from selected workers under a limited budget, improving the number over all spatial crowdsourcing tasks. Besides, Zhao et al. [30] thought that the quality of task accomplishment is mostly related to the worker's preference for the task category.

In this paper, to improve the performance of task assignment, we present a novel, efficient, and flexible approach by jointly considering multiattributes and preferences.

\section{Problem Definition}

In this section, we introduce some basic concepts of spatial crowdsourcing task assignment and then give the formal definitions. For the convenience of the following description, we firstly list the symbols used in this paper, see Table 1.

We consider there are a set of workers $W=\left\{w_{1}, w_{2}, w_{i}, \ldots, w_{m}\right\}$ and a set of tasks $T=\left\{t_{1}, t_{2}, t_{j}, \ldots, t_{n}\right\}$. Subscripts $i$ and $j$ are the worker ID and task ID, respectively. A worker $w_{i}$ is represented as a tuple of the form $\left\langle l^{i}, r^{i}, q^{i}\right\rangle$, where $l^{i}=\left\langle\operatorname{lon}^{i}, \mid a^{i}\right\rangle$ is the location of the work $i, \operatorname{lon}^{i}$ and lat ${ }^{i}$ are the longitude and latitude of the worker $i$, respectively, and $r^{i}$ represents the reputation of the worker $i$ and $q^{i}$ is the task quota of the worker $i$. A spatial crowdsourcing task $t_{j}$ is represented as a tuple of the form, i.e. $t_{j}=\left\langle l^{j}, R^{j}, B^{j}\right\rangle$, where $l^{j}=\left\langle\operatorname{lon}^{j}, \operatorname{lat}^{j}\right\rangle$ is the location of the task $j$, which is represented by a longitude-latitude coordinate, $\operatorname{lon}^{j}$ and lat ${ }^{j}$ are the longitude and latitude of the task $j$, respectively, and $B^{j} \in \mathbb{R}^{+}$is the limited budget of the task $j$.

Definition 1 (decision matrix for a task). Given a set of tasks $T$ and a set of workers $W$. Let $W^{j}$ be the set of workers within the region of the radius $R$ of the task $t_{j} \in T$ and $f$ be the number of attributes that are considered when assigning tasks. To facilitate the description of algorithms subsequently, the task and the worker are attached to the matrix columns. Then, the decision matrix of the task $t_{j}$ is shaped as $D M_{n \times(f+2)}^{j}$, where $n=\left|W^{j}\right|$.

For example, the worker $w_{i} \in W^{j}$; the attributes involved are the distance and workers' reputation; then, the item $s^{j}=$ $\left\langle j, i, d_{j i}, r^{i}\right\rangle$ is included in $D M^{j}$ and $d_{j i}$ represents the distance between $t_{j}$ and $w_{i}$.

Setting the radius value of a task is one of the central aspects of task assignment. A very low radius value would result in a low task completion rate because of the lack of enough workers. In contrast, a very high radius value would have no practical significance because of the unavailability of workers willing to travel a long distance to perform a task. Previous studies suggest that the most acceptable distance for the workers is $0-2 \mathrm{~km}[1,7,13]$. In practice, some workers may be tempted by the larger budget to perform remote tasks. In this paper, we assume (1) a task with a higher budget can select workers from a wider region and (2) some workers are willing to travel further for the higher rewards. So, the radius $R^{j}$ is positively affected by the budget $B^{j}$ and negatively affected by the extra allowance per kilometer $\beta$. Let a worker's accepted baseline distance be $\gamma$ in the condition of a baseline payment $P$ for a task. When a task's budget is less than the baseline payment $P$, the task is not likely to be accepted by any worker; at this situation, the radius is represented by a negative number, because no workers locate within a region of a task's negative radius. Then, the method in [16] is slightly changed to compute $R^{j}$ :

$$
R^{j}= \begin{cases}\gamma+\frac{B^{j}-P}{\beta}, & B^{j} \geq P, \\ -1, & B^{j}<P .\end{cases}
$$

Identifying the value of the parameter $P$ is not our emphasis. We focus on proposing a flexible spatial task assignment method that considers multiple attributes affecting the result in the task assignment and different requirement preferences for each attribute. Hence, we set $P$ as the lowest budget among the budgets of all tasks.

Our goal is to select the worker with the highest rating of combined distance and reputation for a task. However, if the above decision matrix is directly used to determine the task 
TABLE 1: List of symbols.

\begin{tabular}{lc}
\hline Symbol & Meaning \\
\hline$W$ & The set of workers, $W=\left\{w_{1}, w_{2}, \ldots w_{i}, \ldots, w_{m}\right\}$ \\
$T$ & The set of tasks, $T=\left\{t_{1}, t_{2}, \ldots t_{j}, \ldots, t_{n}\right\}$ \\
$i$ & A worker's number, $i \in\{1,2, \ldots, m\}$ \\
$j$ & A spatial task's number, $j \in\{1,2, \ldots, n\}$ \\
$l^{i}$ & The location of the worker, $i$ \\
$l^{j}$ & The location of the task, $j$ \\
$B^{j}$ & The budget of the task, $j$ \\
$R^{j}$ & The radius of the task, $j$ \\
$r^{i}$ & The reputation of the worker, $i$ \\
$d_{j i}$ & The distance from the task $j$ to the worker, $i$ \\
$\beta^{j}$ & The extra allowance per kilometer \\
$P$ & Worker's accepted baseline distance \\
$D M^{j}$ & The baseline payment of a task \\
$E^{j}$ & The decision matrix of the task, $j$ \\
$p^{j}$ & The extra allowance for the task, $j$ \\
$a^{i}$ & The reward for the task, $j$ \\
$q^{i}$ & The amount of task assigned to the worker, $i$ \\
$S$ & The task quota of the worker, $i$
\end{tabular}

assignment, there are two problems. Firstly, the orders of magnitude of attributes are usually different, owing to the different natures of attributes. If the original value is directly used for rating the items, the role of the attribute with the higher value in the comprehensive rating will be highlighted, and the role of the attribute with the lower value will be relatively weakened. Secondly, the distance is a cost-type attribute and the reputation is a benefit-type attribute, which means the distance and the reputation have different influence trend on ratings. Therefore, in order to ensure the reliability of the rating results, it is necessary to normalize the original data. We adopt the linear proportional transformation method to normalize the distance and reputation, as shown in the following equations:

$$
\begin{aligned}
& d_{j i}= \begin{cases}\frac{\max _{i \in W^{j}} d_{j i}-d_{j i}}{\max _{i \in W^{j}} d_{j i}-\min _{i \in W^{j}} d_{j i},} & \max _{i \in W^{j}} d_{j i} \neq \min _{i \in W^{j}} d_{j i}, \\
1, & \text { otherwise, }\end{cases} \\
& r^{i}= \begin{cases}\frac{r^{i}-\min _{i \in W^{j}} r^{i}}{\max _{i \in W^{j}} r^{i}-\min _{i \in W^{j}} r^{i}}, & \max _{i \in W^{j}} r^{i} \neq \min _{i \in W^{j}} r^{i} \\
1, & \text { otherwise. }\end{cases}
\end{aligned}
$$

Definition 2 (reward for a task). When a task is completed, the requester must offer rewards to the corresponding worker. Let $E^{j}$ represent the extra allowance for the task $j$ when it is completed by a worker without $\gamma$. Then, the reward of the task $j$ is expressed in the following equation:

$$
p^{j}=P+E^{j}
$$

where $E^{j}$ is related to the parameter $\beta, d_{j i}, \gamma$. The farther a worker travels, the more extra allowance he should get. If the worker $w_{i}$ completes the task $t_{j}$ within the accepted baseline distance $\gamma$, then $E^{j}$ equals to 0 ; otherwise, $E^{j}$ is proportional to the extra distance and the extra allowance, so $E^{j}$ is computed in the following equation [16]:

$$
E^{j}= \begin{cases}\beta\left(d_{j i}-\gamma\right), & d_{j i}>\gamma \\ 0, & d_{j i} \leq \gamma\end{cases}
$$

The parameter $d_{j i}$ mentioned above involves the location of the task and the worker. We computed $d_{j i}$ from the task $t_{j}$ to the worker $w_{i}$ by the Haversine formula [31]:

$$
d_{j i}=R \times 2 \times \arcsin \left(\left(\sin ^{2}\left(\frac{\mathrm{lat}^{j}-\mathrm{lat}^{i}}{2}\right)+\cos \left(\mathrm{lat}^{j}\right) \times \cos \left(\operatorname{lat}^{i}\right) \times \sin ^{2}\left(\frac{\operatorname{lon}^{j}-\operatorname{lon}^{i}}{2}\right)\right)^{1 / 2}\right)
$$

where $R$ refers to the earth's radius [12].

Definition 3 (TASC-MADM problem). Given a set of tasks $T$ and a set of workers $W$, we assume that each task is assigned to the optimal worker. Let $S=\left\{s^{1}, s^{2}, \ldots, s^{j}, \ldots, s^{n}\right\}$ represent the selected workers for all tasks, $s^{j}=\left\langle j, i, d_{j i}, r^{i}\right\rangle$, $s^{j} \in D M^{j}$, and TASC-MADM is to find $s^{j}$ such that the following linear combination is optimized: 


$$
\max _{i \in W^{j}}\left(\operatorname{score}_{j i}\right)=\max _{i \in W^{j}}\left(w_{0} \times d_{j i}+w_{1} \times r^{i}\right),
$$

where $j$ and $i$ are the matching task-worker pair; and $w_{v} \in[0,1]$ is the attribute importance parameter, $\sum_{v \in\{0,1\}} w_{v}=1$.

The weights are usually determined objectively or subjectively. The entropy method is generally used to objectively get weighting of every attribute [32]. However, we hope that the task assignment operation can set the attribute weight to achieve different optimization goals. So, the weight values are set according to the requirement preference of the task for attributes. If a task has more requirement preference for distance more than that for reputation, it can set $w_{0}>0.5$. Otherwise, $w_{0}<0.5$. The setting, $w_{0}=0.5$, implies the following situation: among the workers with the same reputation, the worker with a shorter distance has the priority to be selected. Similarly, among the workers with the same distance, the worker with a higher reputation has the priority to be selected.

3.1. Complexity Analysis. One task assignment of a single task is to find the best worker among $m$ workers; it needs to repeat the assignment $n \times m$ times to complete all task assignments, so the problem is solvable in polynomial time.

\section{Assignment Protocol}

We assume that the workers querying the tasks are willing to accept the tasks. Thus, assigning a task to a worker means selecting the best worker with the highest comprehensive rating of distance and reputation. In this section, we will elaborate on our spatial task assignment algorithm.

4.1. Preparing Decision Matrix. Preparing the task's decision matrix involves two steps. Firstly, we obtain the decision matrix $D M^{j}$ for the task $j$. Each item of the decision matrix represents a candidate worker. In this paper, the attributes, affecting the result of a task assignment, include the reputation of a worker and the distance from a task to a worker. The workers within the radius of the task $j$ are a part of the task's candidates, excluding the workers who are assigned tasks more than their quota (Line 2-4). Secondly, normalize the decision matrix $D M^{j}$ by equations (2) and (3) (Line 5). The pseudocode, obtaining and normalizing the decision matrix $D M^{j}$ for the task $j$, is given by Algorithm 1 .

The computational complexity of Algorithm 1 depends on the loop operation and the normalization operation. The complexities of these two operations are $O(m)$ and $O\left(\left|W^{j}\right|\right)$, respectively. Since $\left|W^{j}\right|$ is usually much less than $|m|$, the total computational complexity is $O(m)$.

4.2. TASC-MADM Approach. As mentioned in Section 3, a spatial task should be assigned to the worker with the highest rating, which optimizes the linear combination of the distance and the reputation.
Algorithm 2 is the pseudocode of the allocation method, namely, TASC-MADM algorithm, which inputs a set of workers $W$, a set of tasks $T$, and the parameters $P, \beta, \gamma$ and returns the best assignment result $S$, containing task-andworker assignment with the highest rating.

Initially, $S$ is set to empty (Line 1), $\left.a^{i}\right|_{i=\{1,2, \ldots, m\}}=0$ (i.e., each worker has been assigned zero times) (Line 2). Next, for each task $t_{j}$, calculate the radius and the distance to the workers and obtain and normalize the decision matrix $D M^{j}$ by calling Algorithm 1 (Line 6). Next, if the decision matrix has more than zero items, compute the scores of items (Line 9) and, simultaneously, compute the reward $p^{j}$ paid by the task $t_{j}$ to the worker $w_{i}$ (Line 10). For subsequent easy operation, the item's score and the reward are associated with other information including the task number, the worker number, the distance, and the reputation (Line 11). Next, we sort the items descending by scores (Line 12). Intuitively, the item with a higher rank indicates a better assignment than other assignments. Finally, the task $t_{j}$ is assigned to the topmost worker (Line 13-17).

The assignment iterates for $n$ rounds (Line 3 ) and finally returns the assignment result of all tasks (Line 18). In each iteration, the computational complexity is $O(m)$, so the total computational complexity is $O(n \times m)$.

\section{Experiment Evaluation}

In this section, we tested the performance of our approach on both real and synthetic data.

5.1. Metrics. In the experiments, we measured the performance of each approach according to the following metrics [23]:

(1) Average task radius $(\delta)$ : this metric measures the average spatial region size of the tasks, which is computed as the average radius of all tasks.

$$
\beta=\frac{1}{|T|} \sum_{j} R^{j} .
$$

(2) Task assignment rate $(\eta)$ : this metric measures the algorithm's effectiveness in assigning several tasks successfully. The task assignment rate $\eta$ is the percentage of assigned tasks among the total amounts of crowded spatial tasks.

$$
\eta=\frac{|S|}{|T|} .
$$

(3) Average reputation of workers assigned tasks ( $\psi)$ : the quality of crowdsourcing result is determined by the worker's quality, which is modeled by the worker's reputation. So, this metric evaluates the quality of tasks completed. It is computed as the total reputation of selected workers divided by the number of selected workers. 
Input: a set $W$, the task $j$

Output: the normalized decision matrix $D M^{j}$ for the task $j$

(1) $D M^{j}=$

(2) For each $w_{i} \in W$ :

(3) If $d_{j i} \leq R^{j}$ and $a^{i}<q^{i}$ :

(4) $s_{i}^{j}=\left\langle j, i, d_{j i}, r^{i}\right\rangle, D M^{j} \leftarrow D M^{j} \cup\left\{s_{i}^{j}\right\}$

(5) Normalize $D M^{j}$ by equations (2) and (3)

(6) Return $D M^{j}$

Algorithm 1: Obtain the decision matrix for a task.

Input: a set of workers $W$, a set of tasks $T$, parameters $P, \beta, \gamma$

Output: $S$, which is the result of task assignment

(1) $S=$

(2) $a^{i}=0, i \in\{1,2, \ldots, m\}$

(3) For each $t_{j} \in T$ :

(4) Calculate the radius $R^{j}$

(5) Calculate $d_{j i}$ from the task $j$ to each worker $i$

(6) Obtain and normalize the decision matrix $D M^{j}$ by calling Algorithm 1

(7) If $\left|D M^{j}\right|>0$ :

(8) For $i^{\prime}$ in range $\left(\left|D M^{j}\right|\right)$ :

(9) Compute the score ${ }_{j i}$ using equation (7)//i $=D M^{j}[i \prime] . i$, it is the number of the $i^{\prime}$ th worker for the task $t_{j}$.

(10) Compute the reward $p^{j}$ using equation (4).

(11) $\quad s_{i}^{j}=s_{i}^{j}$.append $\left(\left\langle p^{j}\right.\right.$, score $\left.\left._{j i}\right\rangle\right)\left(\right.$ i.e. $s_{i}^{j}=\left\langle j, i, d_{j i}, r^{i}, p^{j}\right.$, score $\left.\left._{j i}\right\rangle\right)$

(12) Sorted $D M^{j}$ descending by scores

(13) For each item in $D M^{j}$ :

(14) If $j$ in item:

(15) $\quad s^{j}=$ item, $a^{i}+=1$

(16) $\quad S \leftarrow S \cup\left\{s^{j}\right\}$

(17) Break

(18) Return $S$

Algorithm 2: TASC-MADM algorithm.

$$
\psi=\frac{1}{|S|} \sum_{i \in S} r^{i}
$$

(4) Average distance traveled ( $)$ : this metric measures the travel cost for workers when they complete the assigned tasks; it is computed as the average distance traveled by all the selected workers.

$$
\varsigma=\frac{1}{|S|} \sum_{\langle j, i\rangle \in S} d_{j i} \text {. }
$$

(5) Average budget utilization rate $(\phi)$ : this metric is the average of the budget utilization for all assigned tasks. The budget utilization rate of each assigned task is the ratio of the actual reward paid for the task to the budget of that task.

$$
\phi=\frac{1}{|S|} \sum_{j \in S} \frac{p^{j}}{B^{j}} .
$$

(6) Average reward $(\omega)$ : this is the ratio of the total reward for all the assigned tasks to the number of assigned tasks.

$$
\omega=\frac{1}{|S|} \sum_{j \in S} p^{j} .
$$

The parameter $\delta$ is used to demonstrate the changing of other metrics along with the average radius. The workers' perspective would prefer to keep $\varsigma$ as low as possible. The task requesters' perspective would prefer higher values of $\eta$ and $\psi$, but lower values of $\phi$ and $\omega$.

\subsection{Experimental Setting}

\subsubsection{Datasets}

(i) Real Dataset. We used a real dataset [33] from a crowdsourcing event by taking photos with the location information for 1877 workers and 835 tasks. These tasks and workers were mainly obtained from four cities in China: Foshan, Guangzhou, Shenzhen, and Dongguan.

(ii) Synthetic Dataset. The synthetic data were obtained from a single day dataset from Gowalla in 2010, which included 10956 tasks and 5087 workers 
located in America. This dataset contains four attributes as follows: task ID, task's location, worker ID, and worker's location. Reputations are generated following the uniform distribution of $0 \sim 20000$. Budgets are generated following the uniform distribution of $65 \sim 85$. The task quotas of workers are generated following the uniform distribution of $5 \sim 10$.

5.2.2. Compared Algorithms. The RB-TPSC and BudgetTASC algorithms were selected as the baseline algorithms because they are most closely related to TASC-MADM.

(i) RB-TPSC is a task package assignment method, which aims at maximizing the number of tasks assigned within budget constraints. Quality of results and travel costs are also being considered.

(ii) Budget-TASC is a budget-aware spatial crowdsourcing task assignment method, which aims in maximizing the total quality of tasks completed within budget constraints.

We compared our TASC-MADM with RB-TPSC and Budget-TASC on both real and synthetic datasets.

5.3. Results on Real Dataset. The first three experiments compared TASC-MADM and RB-TPSC under different settings of parameters: $\beta, \gamma$, and $B^{j}$. The results are shown in Figures 1-3. For each parameter, the experiment evaluated the average metrics of the two algorithms under 21 different settings. We set the lowest budget of all tasks as $P=65$. Moreover, for the fairness in considering the effect of distance and reputation, the weight $w_{0}=0.5$.

First, we compared TASC-MADM and RB-TPSC under different $\beta$ values. The results are shown in Figure 1, where the extra allowance per kilometer $\beta$ varies from $0 \sim 20$ monetary units in 1-unit increments, $\gamma=0.5$, and $B^{j}$ is obtained from the dataset. The horizontal axis represents the different settings of $\beta$, while the vertical axis represents the different values of the first six metrics. As can be seen, the average task radius $\delta$ is affected by the extra allowance per kilometer $\beta$ (Figure $1(\mathrm{a})$ ). $\delta$ is maximized when $\beta=1$, increases with $\beta$ if $\beta<1$, and decreases with $\beta$ if $\beta>1$. The change in the trend of other metrics is consistent with the average task radius. This is because, in a region with a smaller radius, it is usually impossible to find enough workers with high reputations. Thus, other metrics decrease with the average radius. Compared with RB-TPSC, TASC-MADM ensures a high average task assignment rate (Figure 1(b)), but reduces the average travel cost of workers (Figure 1(d)) and saves the budget (Figures 1(e) and 1(f)). Besides, our result shows that the average reputation of workers decreases with the average radius (Figure $1(\mathrm{c})$ ), which is more realistic because there are fewer workers to choose from.

Secondly, we compared TASC-MADM and RB-TPSC under different $\gamma$ values. Figure 2 depicts the trend in which the above six metrics change when $\gamma$, the accepted distances without extra remote allowance, varies from $0 \sim 2 \mathrm{~km}$, where $\beta=2$, and $B^{j}$ is obtained from the real dataset. If $\gamma>0.5$, the average radius increases with $\gamma$ and positively affects the abovementioned metrics, except for $\phi$ and $\omega$. Our proposed method achieves a task assignment rate value of 98.56 (Figure 2(b)), but the maximum of the average distance traveled the average reward, and the average budget utilization rates are $\varsigma=1.03 \mathrm{~km}, \omega=65.33$, and $\phi=94.93$, respectively (Figures 2(d)-2(f)). Compared to the RB-TPSC method, our method greatly decreases the average distance traveled by the workers and the average reward offered by the tasks' requester, while maintaining an equally high or a greater average task assignment rate. Moreover, it significantly improves the quality of crowdsourcing results because of the average reputation value of the selected workers increasing by about $250 \%$ (Figure 2 (c)).

The third experiment compared TASC-MADM and RBTPSC under different budgets $(B)$. Figure 3 depicts how the first six metrics change while the tasks' budget $(B)$ varies from $90 \%$ to $110 \%$ in $1 \%$ increments, where $\beta=2, \gamma=0.5$. Since the task with a greater budget should have more workers to choose from, the average radius of the tasks is positively affected by the tasks' budget (Figure 3(a)). As the task radius increases further, the task assignment rate increases to $99.5 \%$ (Figure 3(b)). Compared to the RB-TPSC method, our method decreases the average distance traveled from a range of $1.4 \sim 2.5 \mathrm{~km}$ to a range of $0.8 \sim 1.5 \mathrm{~km}$ (Figure 3(d)), but increases the average reputation by about $147 \%$ (Figure 3(c)), and greatly decreases the average budget utilization rate (Figure $3(\mathrm{e})$ ) and the average reward ((Figure 3(f))). More importantly, with our method, the greater the number of candidate workers, the lower the average reward offered by the requester, and the higher is the quality of the crowdsourcing result. In contrast, RB-TPSC increases the average reward continuously and decreases the average reputation to a stable value of around 500 .

The fourth experiment compared TASC-MADM with RB-TPSC and Budget-TASC when radiuses were changed. For TASC-MADM and RB-TPSC, we selected the average metrics of different $\gamma$ values. In the above experiment, the computed radius belongs to the interval [0,12], when $\gamma$ varied from $0 \sim 2 \mathrm{~km}$ and other parameters were fixed. We have experimented with Budget-TASC in different radiuses varying from $0 \sim 12 \mathrm{~km}$ and then get the average of each metric. For the Budget-TASC, the other parameters were set as follows: $D_{C}$ is set as the earth's radius [10], $P_{L}=0, P_{H}$ is set as the budget of the task, and $P_{M}$ is set as the half of the budget of the task. Because we scaled the worker's reputation to the interval $[0,1], T h_{H M}=0.75, T h_{M L}=0.5$.

The results are shown in Table 2. TASC-MADM outperforms the baseline algorithms in all metrics, except the average reputation. For Budget-TASC, $P_{H}$ is set as the task's total budget, which limits the task to high-quality workers, but also increases the average budget utilized.

5.4. Results on Synthetic Dataset. We repeated the first three experiments in Section 5.3 on the synthetic dataset. Figures 4-6 illustrate the results of TASC-MADM and RBTPSC. Compared with RB-TPSC, our algorithm greatly 


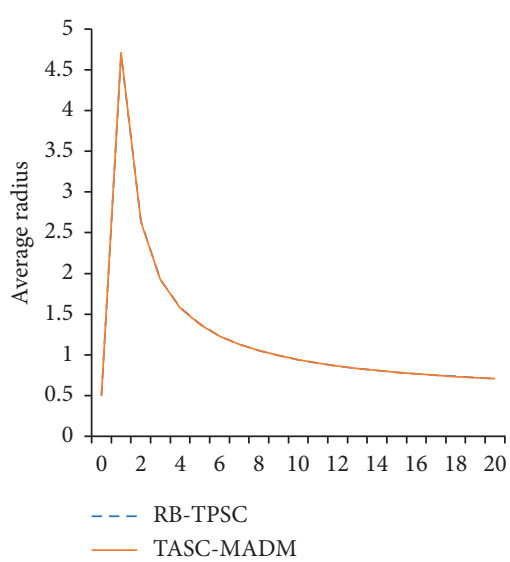

(a)

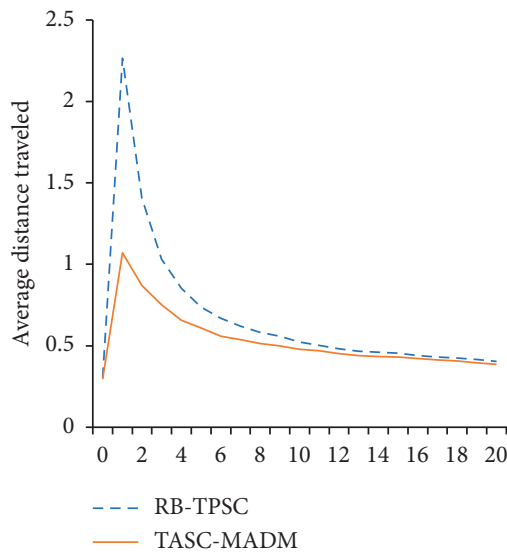

(d)

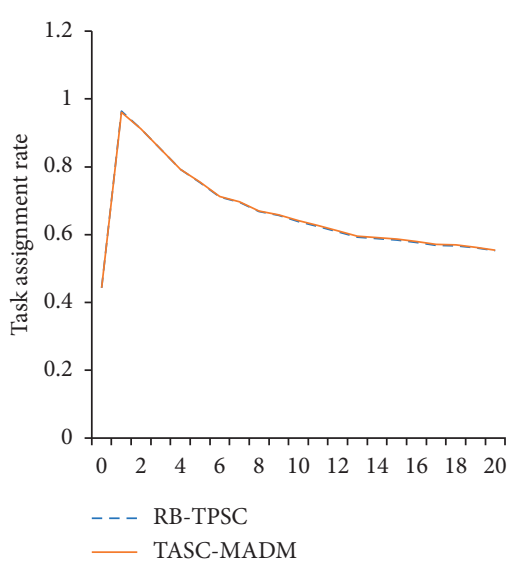

(b)

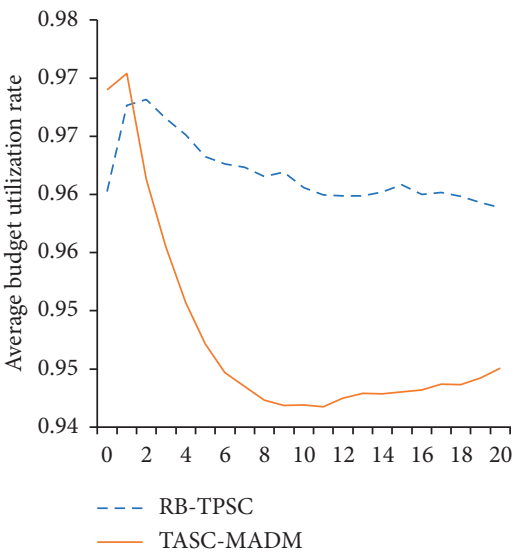

(e)

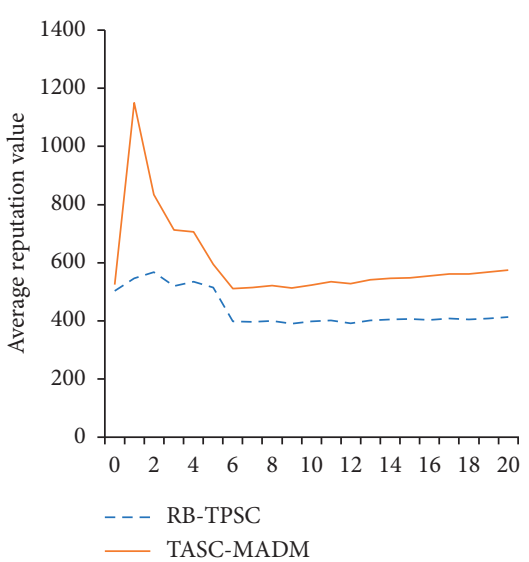

(c)

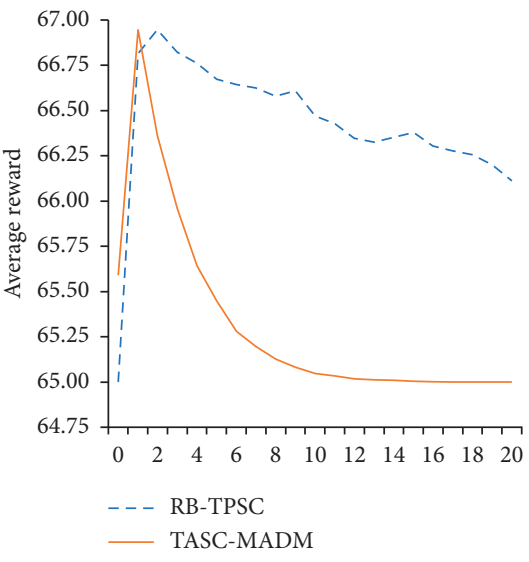

(f)

Figure 1: Performance with an extra allowance per kilometer $(\beta)$ (monetary unit), real dataset.

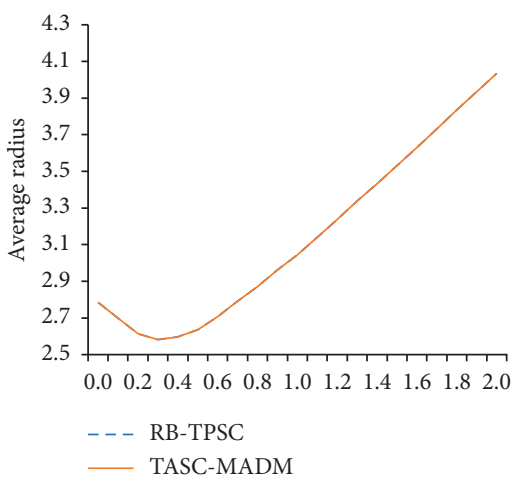

(a)

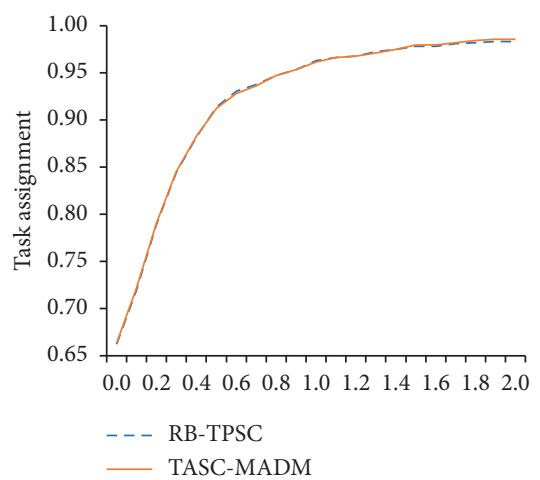

(b)

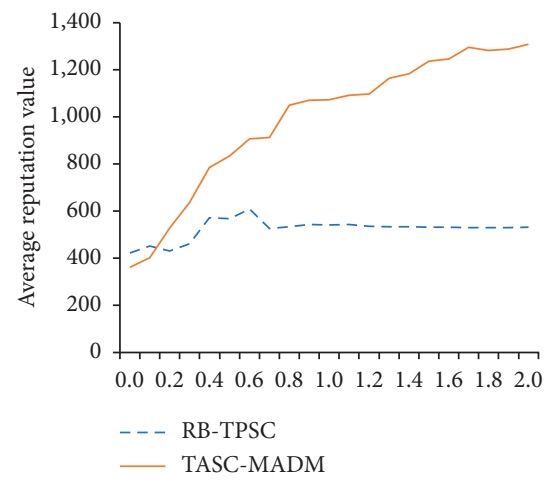

(c)

Figure 2: Continued. 


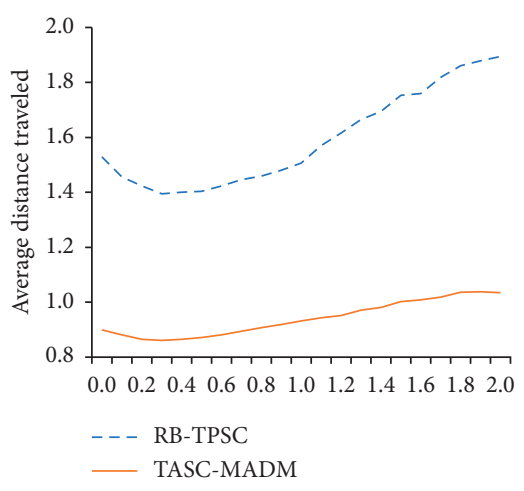

(d)

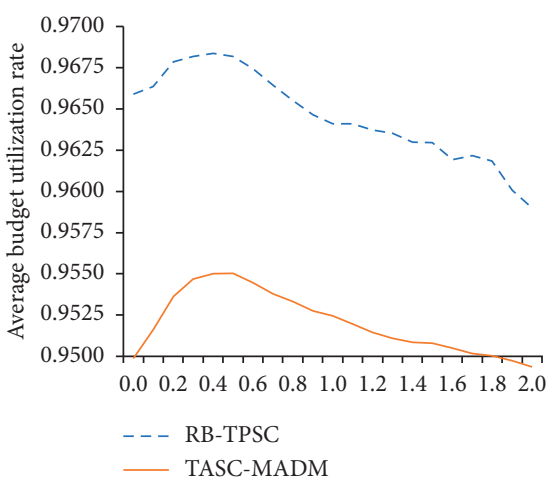

(e)

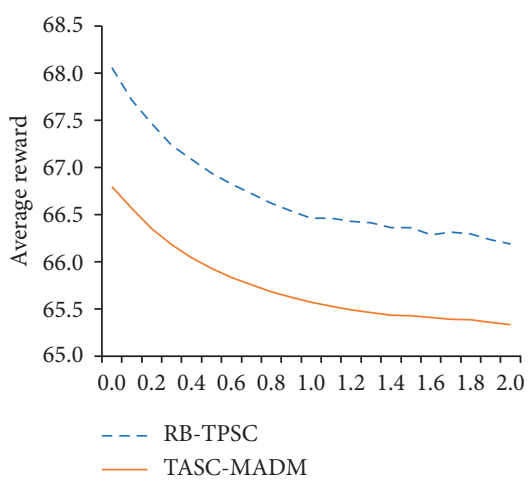

(f)

Figure 2: Performance with different accepted distances without extra remote allowance $(\gamma)(\mathrm{km})$, real dataset.

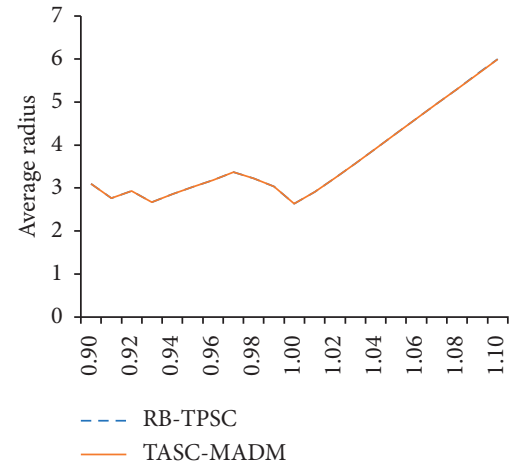

(a)

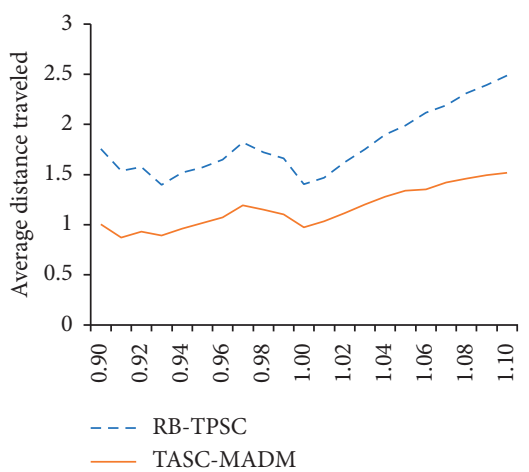

(d)

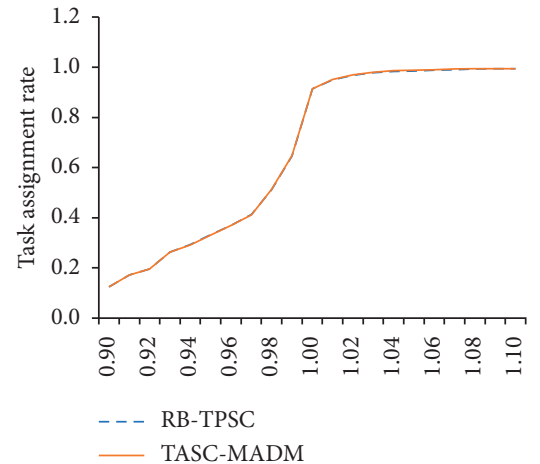

(b)

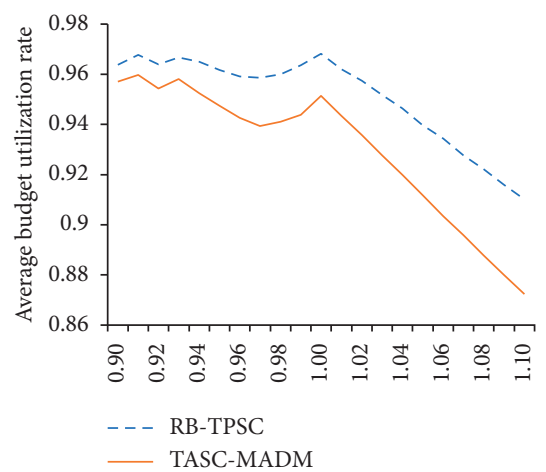

(e)

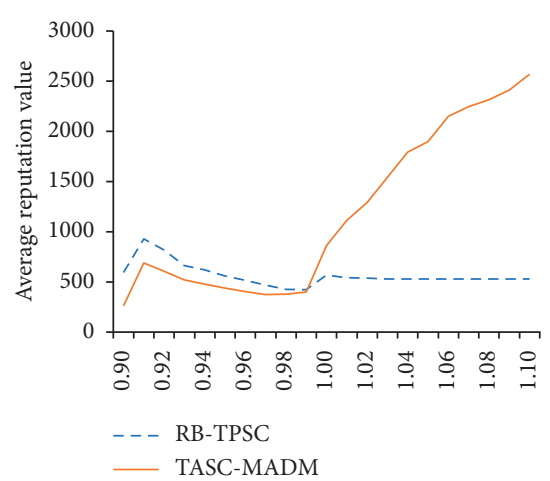

(c)

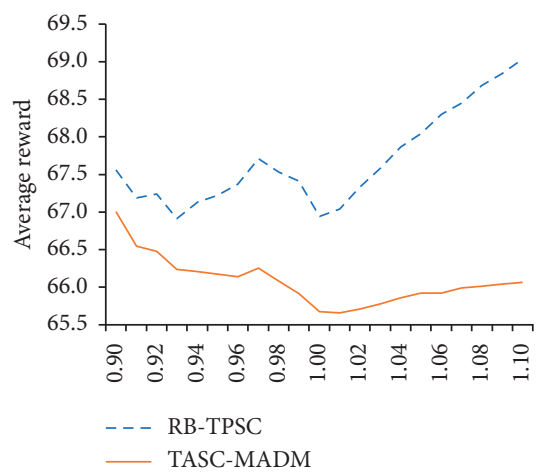

(f)

Figure 3: Performance with an amplitude of variation in budget $(B)$, real dataset.

TABLE 2: Results under different approaches, real dataset.

\begin{tabular}{lccccc}
\hline Algorithms & $\eta(\%)$ & $\psi$ & $\zeta$ & $\phi$ & $\omega$ \\
\hline Budget-TASC & 85.67 & 1390.63 & 1.4 & 1 & 77.61 \\
RB-TPSC & 93.95 & 523.39 & 1.59 & 0.9645 & 66.72 \\
TASC-MADM & 94.18 & 987.46 & 0.78 & 0.9509 & 65.98 \\
\hline
\end{tabular}

improves the task assignment rate (Figures 4(b), 5(b), and 6(b)) and the result's quality (Figures 4(c), 5(c), and 6(c)). However, for the synthetic dataset, the task locates in a slightly sparse scenario with few workers. Then, the higher task assignment rate implies the higher travel cost (Figures 4(d), 5(d), and 6(d)). Correspondingly, the budget used is increased (Figures 4(e), 4(f), 5(e), 5(f), 6(e), and $6(\mathrm{f}))$.

Next, based on the synthetic dataset, we compared TASC-MADM with RB-TPSC and Budget-TASC when radiuses were changed. For TASC-MADM and RB-TPSC, we selected the average metrics of different $\gamma$ values; the computed radius approximately belongs to the interval $[6,9]$ 


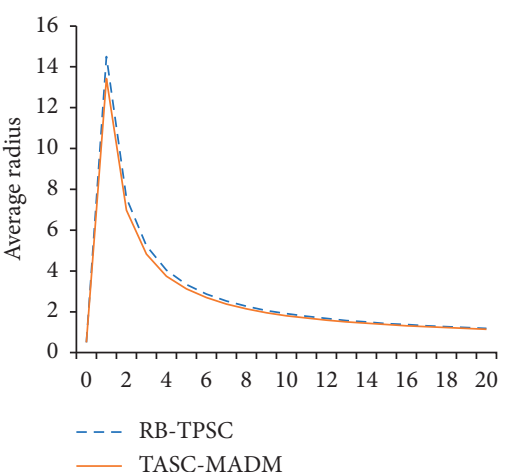

(a)

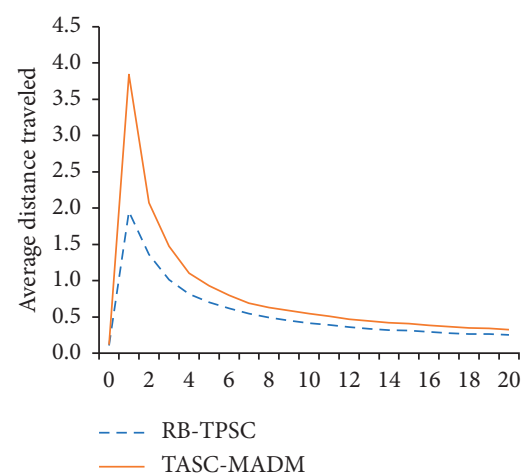

(d)

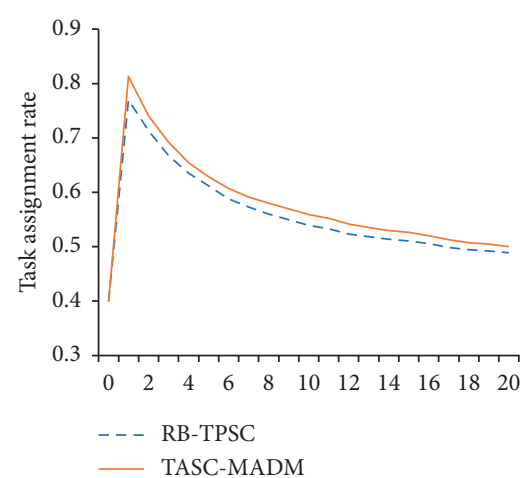

(b)

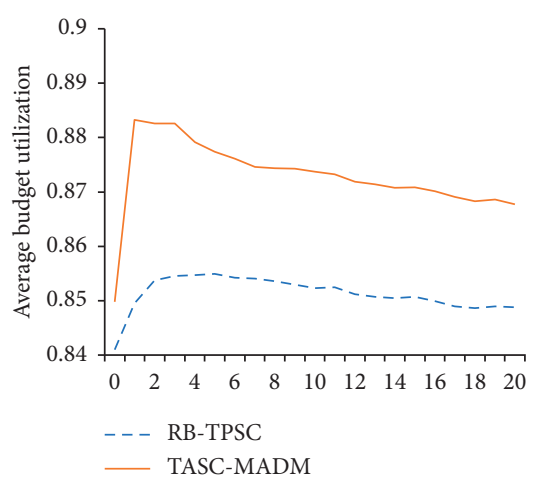

(e)

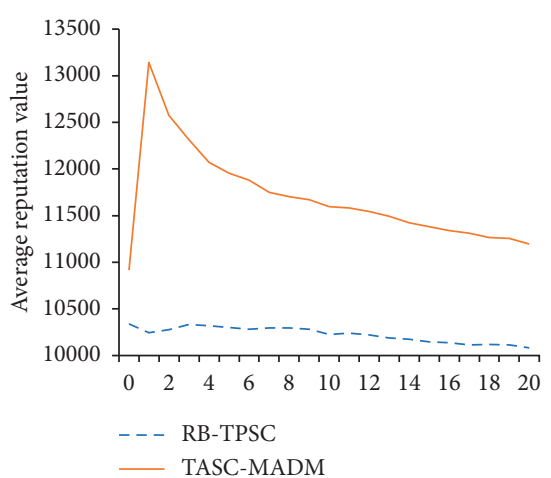

(c)

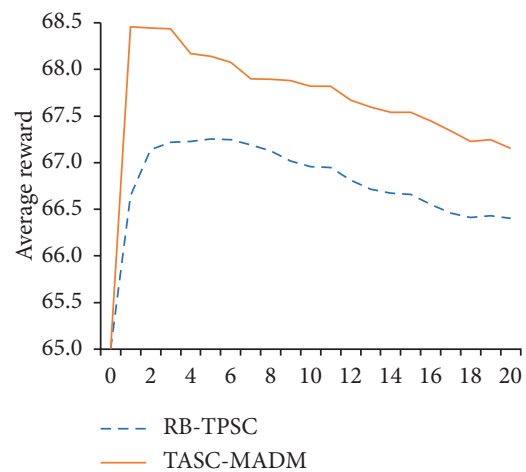

(f)

FIgURe 4: Performance with an extra allowance per kilometer $(\beta)$ (monetary unit), synthetic dataset.

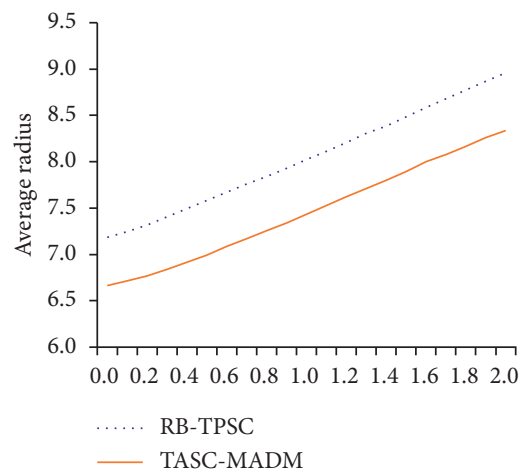

(a)

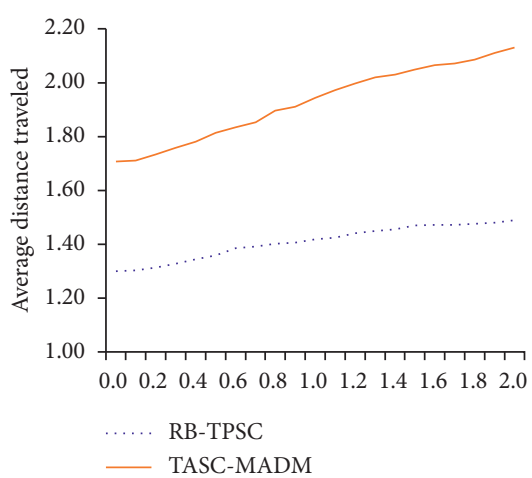

(d)

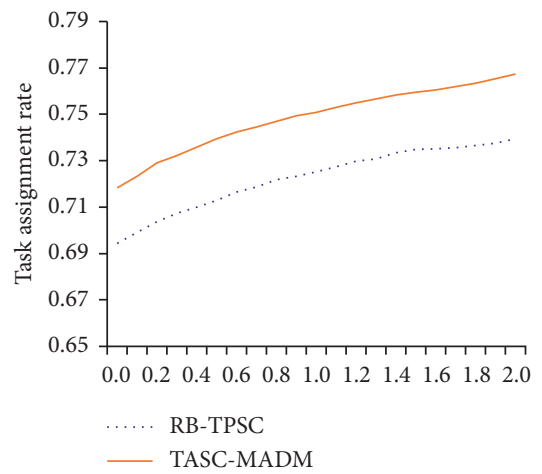

(b)

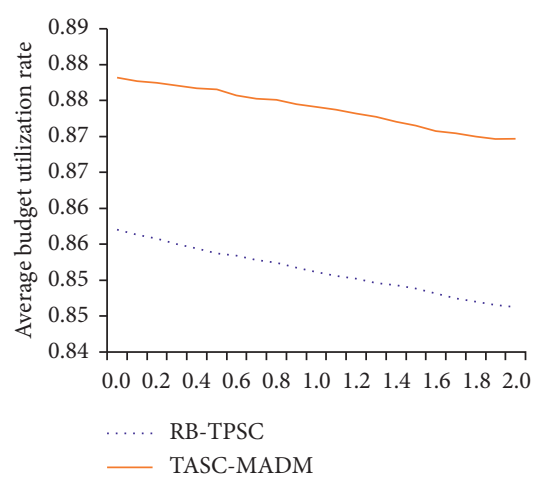

(e)

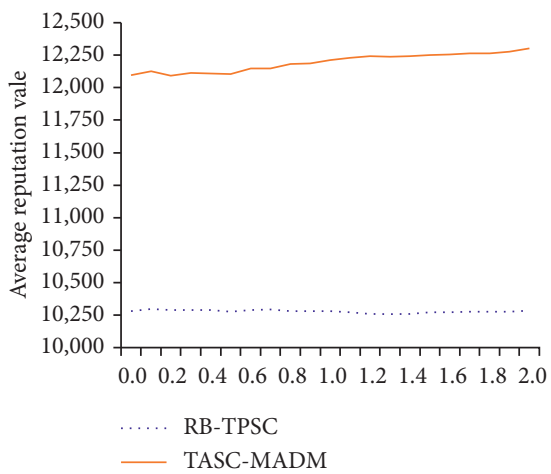

(c)

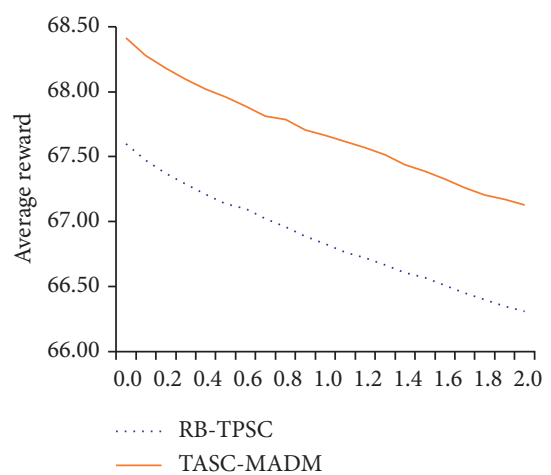

(f)

FIGURE 5: Performance with different accepted distances without extra remote allowance $(\gamma)(\mathrm{km})$, synthetic dataset. 


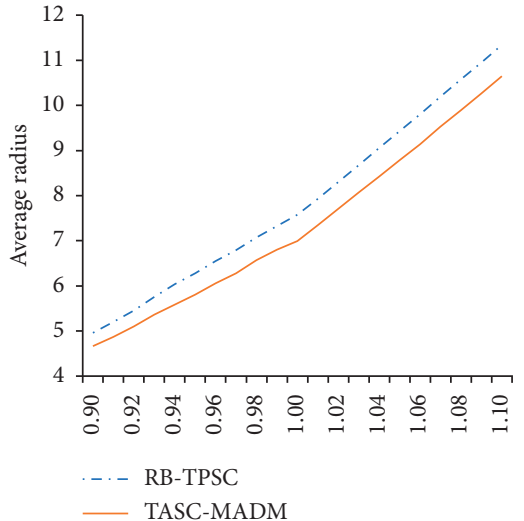

(a)

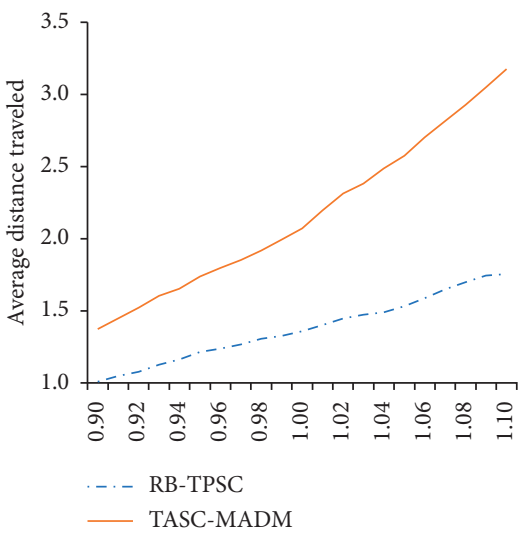

(d)

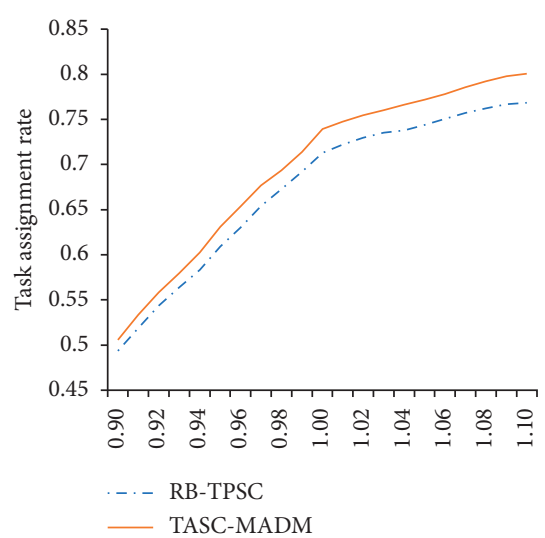

(b)

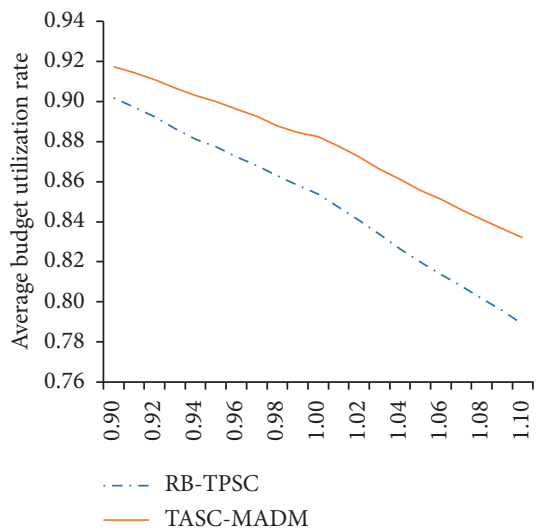

(e)

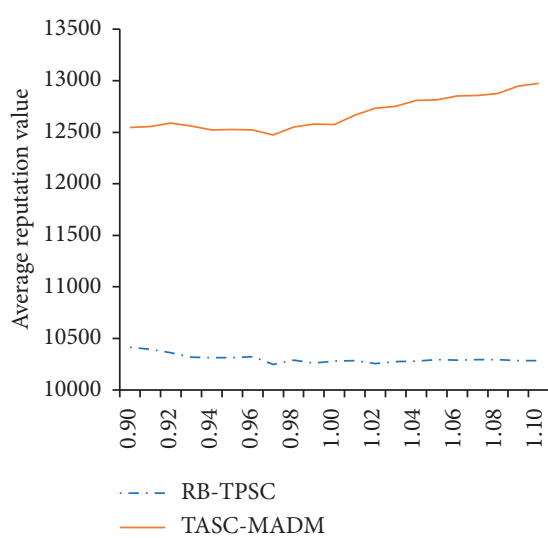

(c)

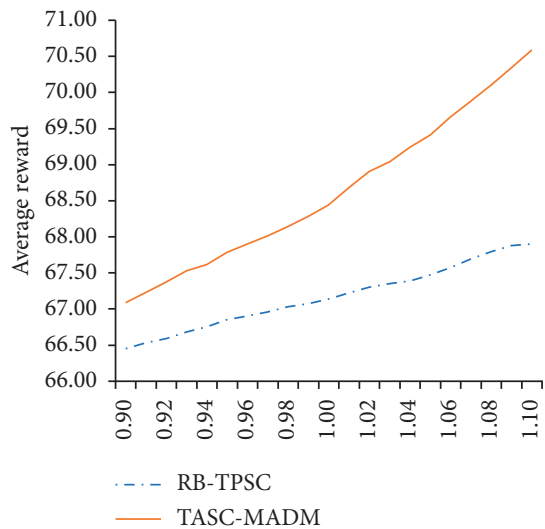

(f)

FIgURe 6: Performance with an amplitude of variation in budget (B), synthetic dataset.

(Figure 5(a)). So, for Budget-TASC, the radius varied from $6 \sim 9 \mathrm{~km}$; other parameters were set as in the fourth experiment.

The experimental results (Table 3) show that TPSC$\mathrm{ADM}$ obtains the highest task assignment rate, but BudgetASC achieves the highest quality and RB-TPSC spends the lowest budget.

5.5. Effect of Task's Attribute Preference. Our approach, TASC-MADM, enables the task to be assigned to satisfy demands for different goals. If the result's quality is the primary goal, the task should select workers with a high reputation. However, if saving cost is the most concerned objective, the worker closer to the task should be chosen first. Figure 7 shows the influence of tasks' attributes preferences on the proposed approach's performance. $w_{0}=0.5$, all targets are considered fairly. $w_{0}<0.5$, the task prefers the worker's reputation to the distance; the result's quality is significantly improved. However, the travel cost and budget utilized are increased. When $w_{0}>0.5$, the travel cost and the budget utilized are saved, whereas the result's quality is decreased. Our approach can maximize task assignment rate by setting $w_{0}=1$, or maximize the quality of workers selected by setting $w_{0}=0$.
5.6. Efficiency of Algorithms. The efficiency of the algorithms is measured in CPU cost. The Budget-TASC algorithm's computational complexity is $O\left(n \times m^{2}\right)$, and that of RBTPSC and TASC-MADM is $O(n \times m)$. we compare TASCMADM with RB-TPSC and Budget-TASC in CPU cost. Each of the programs runs $3 \times 21$ rounds. The average time per round is used to measure the algorithm's efficiency. As shown in Figure 8, our approach significantly improves the efficiency of spatial task assignment.

5.7. Summary of Experiment Results. We summarized the major finding as follows:

(i) If the task is located in a worker-density area, the proposed TASC-MADM approach exhibits better results than RB-TPSC. It also performs better than Budget-TASC on the metrics, except for the quality of workers.

(ii) If the task is located in a worker-sparsity area, the proposed TASC-MADM approach performs better than RB-TPSC in terms of the average assignment rate and quality of workers. But it leads to more travel cost and budget utilized. Budget-TASC obtains the best quality because it considers the quality first. 
TABLE 3: Results under different approaches, synthetic dataset.

\begin{tabular}{|c|c|c|c|c|c|}
\hline Algorithms & $\eta$ & $\psi$ & $\zeta$ & $\phi$ & $\omega$ \\
\hline Budget-TASC & 0.72743975 & 13672.17 & 1.417697 & 1 & 77.58585 \\
\hline RB-TPSC & 0.72253468 & 10278.82 & 1.408169 & 0.851329 & 66.86784 \\
\hline TPSC-ADM & 0.74825306 & 12194.53 & 1.926809 & 0.873916 & 67.68671 \\
\hline
\end{tabular}

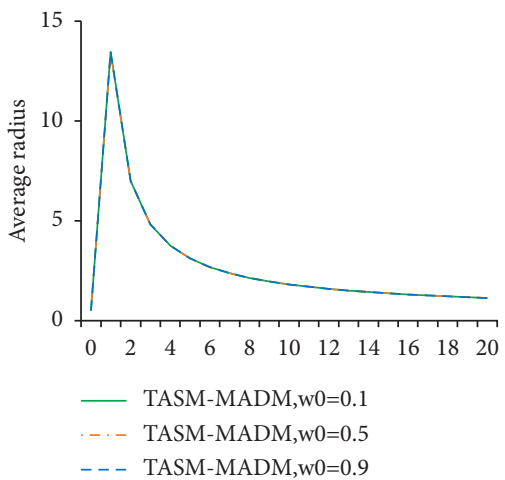

(a)

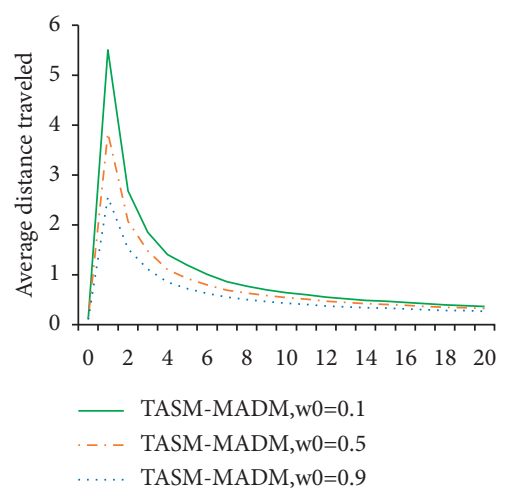

(d)

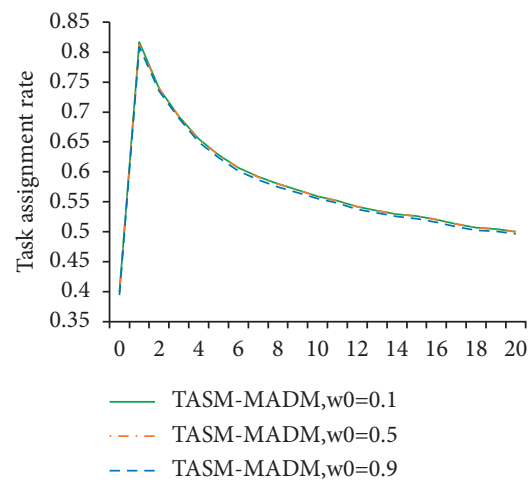

(b)

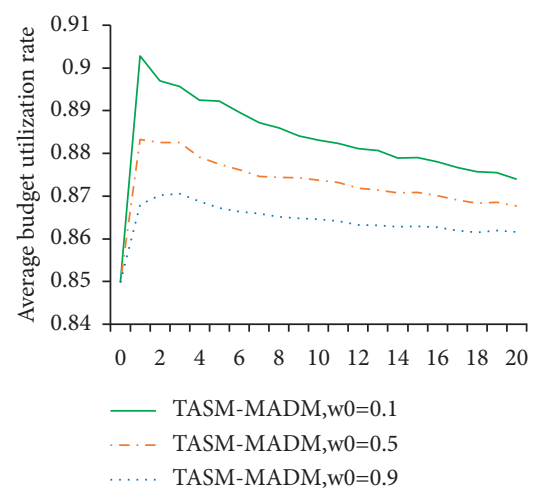

(e)

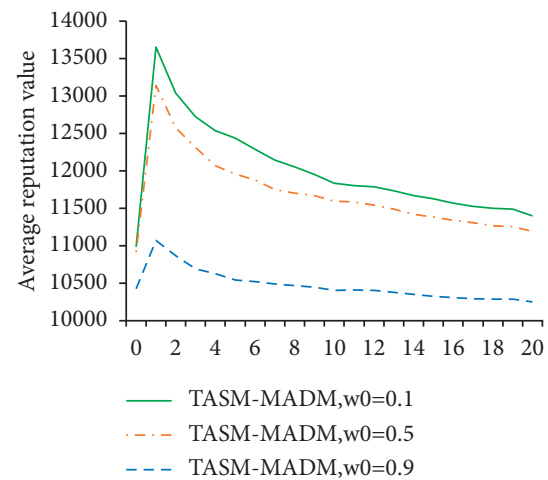

(c)

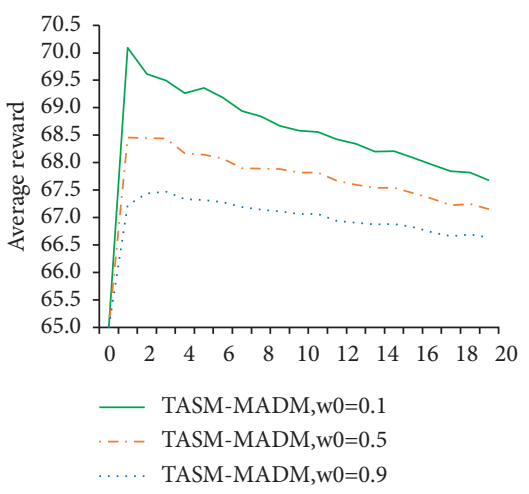

(f)

Figure 7: Effect of tasks' attributes preferences on the TASC-MADM approach. Performance with an extra allowance per kilometer $(\beta)$ (monetary unit), synthetic dataset.

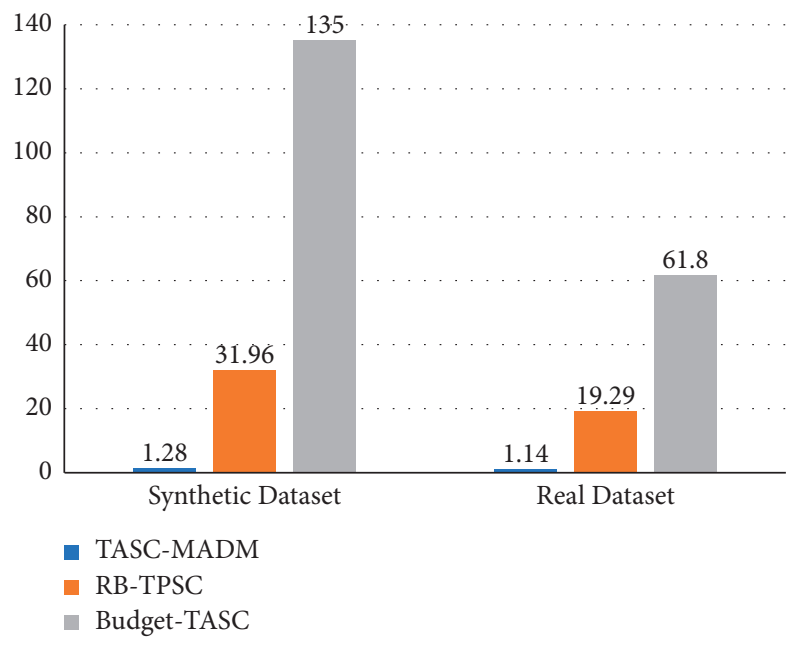

Figure 8: Average CPU cost. 
(iii) In the terms of CPU cost, the proposed TASCMADM approach is superior to the baseline algorithms.

5.8. Discussion. This section discusses the advantages and limitations of the TASC-MADM approach. The advantages are listed as follows:

(1) Effective. The TASC-MADM approach improves the task assignment rate. By setting different attribute weights, it can maximize the task assignment rate or the quality of crowdsourcing results.

(2) Efficient. The TASC-MADM approach enhances efficiency because of computing simply.

(3) Extensible. Theoretically, our method can be extended to solving decision problems involving any number of attributes.

There still exist the following limitations in the TASCMADM approach.

(1) Quality Quantification of Workers. In this paper, the worker quality is modeled as a reputation value, to reflect the quality of crowdsourced results. In practice, the same worker's quality may differ in specific tasks. How to quantify the quality of workers is not covered in this paper.

(2) Efficiency of Indexing Records. The TASC-MADM approach exhaustively searches all the records to identify the candidate of a task, which makes it less efficient to get the decision matrix on large datasets.

\section{Conclusion}

This paper focuses on designing an efficient task assignment approach, which can deal with the situation where tasks have different require preferences for different task attributes, to achieve different goals. Our task assignment approach can be extended to scenarios containing any number of attributes. In addition to the distance and reputation, other criteria such as the workers' skills can be considered.

As for future work, more factors, such as workers' willingness to accept tasks and their quality differences in different professional fields, are included in task assignments. In addition, improving the efficiency of indexing records to make the allocation scheme suitable for large datasets is a valuable research topic.

\section{Data Availability}

The data of allocated sharing tasks are available from China Society for Industrial and Applied Mathematics, 2017, retrieved on September 2, 2020, from http://www.mcm.edu. cn/html_cn/node/460baf68ab0ed0e1e557a0c79b1c4648. html.

\section{Conflicts of Interest}

The authors declare that there are no conflicts of interest regarding the publication of this paper.

\section{Acknowledgments}

The authors thank researchers working at Guangxi Key Laboratory of Trusted Software, especially Xuguang Bao and Manli Zhu, for their suggestion during the research and preparation of the manuscript. This work was funded by Guangxi Key Laboratory of Trusted Software (No. kx201727), Project to Improve the Scientific Research Basic Ability of Middle-Aged and Young Teachers (No. 2019KY0226), Natural Science Foundation of China (Nos. 61966009, U1811264, and U1711263), and Natural Science Foundation of Guangxi Province (Nos. 2019GXNSFBA245049, 2019GXNSFBA245059, and 2018GXNSFDA281045).

\section{References}

[1] L. Kazemi and C. Shahabi, "GeoCrowd: enabling query answering with spatial crowdsourcing. GIS," in Proceedings of the ACM International Symposium on Advances in Geographic Information Systems, pp. 189-198, CA, USA, November 2012.

[2] J. Xiong, M. Zhao, M. Z. A. Bhuiyan, L. Chen, and Y. Tian, "An AI-enabled three-party game framework for guaranteed data privacy in mobile edge crowdsensing of IoT," IEEE Transactions on Industrial Informatics, vol. 17, no. 2, pp. 922-933, 2021.

[3] J. Xiong, R. Ma, L. Chen et al., "A personalized privacy protection framework for mobile crowdsensing in IIoT," IEEE Transactions on Industrial Informatics, vol. 16, no. 6, pp. 4231-4241, 2020.

[4] DiDi, Didi Chuxing Corporate Citizenship Report, https:// www.didiglobal.com/aboutdidi/responsibility, 2017.

[5] J. Xiong, J. Ren, L. Chen et al., "Enhancing privacy and availability for data clustering in intelligent electrical service of IoT," IEEE Internet of Things Journal, vol. 6, no. 2, pp. 1530-1540, 2019.

[6] C. Zhao, P. Cheng, L. Chen, X. Lin, and C. Shahabi, "Fair task assignment in spatial crowdsourcing," Proceedings of the VLDB Endowment, vol. 13, no. 12, pp. 2479-2492, 2020.

[7] M. Venanzi, A. Rogers, and N. R. Jennings, "Crowdsourcing spatial phenomena using trust-based heteroskedastic Gaussian processes," in Proceedings of the First Conference on Human Computation and Crowdsourcing (HCOMP), pp. 182-189, Palm Springs, CA, USA, November 2013.

[8] J. Xiong, R. Bi, M. Zhao, J. Guo, and Q. Yang, "Edge-assisted privacy-preserving raw data sharing framework for connected autonomous vehicles," IEEE Wireless Communications, vol. 27, no. 3, pp. 24-30, 2020.

[9] B. Van, B. V. D. Haak, M. Parks, and M. Castells, "The future of journalism: networked journalism rethinking journalism in the networked digital age," International Journal of Communication, vol. 6, pp. 2923-2938, 2012.

[10] M. Zook, M. Graham, S. Taylor, and S. Gorman, "Volunteered geographic information and crowdsourcing disaster relief: a case study of the Haitian earthquake," World Medical "Health Policy, vol. 2, no. 2, pp. 7-33, 2010.

[11] Y. Tong, L. Chen, and C. Shahabi, "Spatial crowdsourcing: challenges, techniques, and applications," Proceedings of the VLDB Endowment, vol. 10, pp. 1988-1991, 2017.

[12] J. Xiong, X. Chen, Q. Yang, L. Chen, and Z. Yao, "A taskoriented user selection incentive mechanism in edge-aided mobile crowdsensing," IEEE Transactions on Network Science and Engineering, vol. 7, no. 4, pp. 2347-2360, 2020. 
[13] F. Alt, A. Shirazi, A. Schmidt, U. Kramer, and Z. Nawaz, "Location-based crowdsourcing: extending crowdsourcing to the real world," in Proceedings of the 6th Nordic Conference on Human-Computer Interaction, pp. 13-22, Reykjavik, Iceland, October 2010.

[14] L. Kazemi, C. Shahabi, and L. Chen, "GeoTruCrowd: trustworthy query answering with spatial crowdsourcing," in Proceedings of the ACM International Symposium on Advances in Geographic Information Systems, pp. 314-323, Orlando, Florida USA, November 2013.

[15] U. ul Hassan and E. Curry, "Efficient task assignment for spatial crowdsourcing: a combinatorial fractional optimization approach with semi-bandit learning," Expert Systems with Applications, vol. 58, pp. 36-56, 2016.

[16] C. Miao, H. Yu, Z. Shen, and C. Leung, "Balancing quality and budget considerations in mobile crowdsourcing," Decision Support Systems, vol. 90, pp. 56-64, 2016.

[17] P. Wu, E. W. T. Ngai, Y. Wu, and Y. Wu, "Toward a real-time and budget-aware task package allocation in spatial crowdsourcing," Decision Support Systems, vol. 110, pp. 107-117, 2018.

[18] H. To, C. Shahabi, and L. Kazemi, "A server-assigned spatial crowdsourcing framework," ACM Transactions on Spatial Algorithms and Systems, vol. 1, no. 1, pp. 1-28, 2015.

[19] L. T. Thanh, T. D. Huynh, A. Rosenfeld, S. Ramchun, and N. R. Jennings, "BudgetFIx: budget limited crowdsourcing for interdependent task allocation with quality guarantees. 13th international conference on autonomous agents and multiagent systems," AAMAS 2014, vol. 1, pp. 477-484, 2014.

[20] U. Hassan and E. Curry, "A capability requirements approach for predicting worker performance in crowdsourcing," vol. 1, pp. 429-437, in Proceedings of the 2013 9th International Conference on Collaborative Computing: Networking, Applications and Worksharing (CollaborateCom), vol. 1, IEEE Computer Society, Austin, TX, USA, October 2013.

[21] H. To, G. Gabriel, and C. Shahabi, "A framework for protecting worker location privacy in spatial crowdsourcing," Proceedings of the VLDB Endow, vol. 7, no. 10, pp. 919-930, 2014.

[22] U. U. Hassan, S. O'Riain, and E. Curry, E_ects of Expertise Assessment on the Quality of Task Routing in Human Computation, https://doi.org/10.14236/ewic/sohuman2013.2, 2013.

[23] Y. Tong, Z. Zhou, Y. Zeng, L. Chen, and C. Shahabi, "Spatial crowdsourcing: a survey," VLDB JOURNAL, vol. 1, pp. 217250, 2020.

[24] U. U. Hassan and E. Curry, "A multi-armed bandit approach to online spatial task assignment," in Proceedings of the 2014 IEEE 11th Int Conf on Ubiquitous Intelligence and Computing and 2014 IEEE 11th Intl Conf on Autonomic and Trusted Computing and 2014 IEEE 14th Intl Conf on Scalable Computing and Communications and its Associated Workshops, pp. 212-219, IEEE, Bali, Indonesia, December 2014.

[25] Y. Zeng, Y. Tong, L. Chen, and Z. Zhou, "Latency-Oriented task completion via spatial crowdsourcing," in Proceedings of the 2018 IEEE 34th International Conference on Data Engineering (ICDE), pp. 317-328, \{IEEE\} Computer Society, Paris, France, April 2018.

[26] L. Tran, H. To, L. Fan, and C. Shahabi, "A real-time framework for task assignment in h spatial crowdsourcing," ACM Transactions on Intelligent Systems and Technology, vol. 9, no. 3, pp. 1-26, 2018.
[27] Z. Shen, Y. Han, C. Miao, and J. Weng, "Trust-based web service selection in virtual communities," Web Intelligence and Agent Systems, vol. 9, pp. 227-238, 2011.

[28] Y. Han, S. Liu, A. Kot, C. Miao, and C. Leung, "Dynamic witness selection for trustworthy distributed cooperative sensing in cognitive radio networks," in Proceedings of the International Conference on Communication Technology Proceedings, ICCT, pp. 1-6, Baku, Azerbaijan, October 2011.

[29] P. Cheng, X. Lian, Z. Chen et al., "Reliable diversity based spatial crowdsourcing by moving workers," Proceedings of the VLDB Endow, vol. 8, no. 10, pp. 1022-1033, 2015.

[30] Y. Zhao, J. Xia, G. Liu et al., "Preference-aware task assignment in spatial crowdsourcing," in Proceedings of the The Thirty-Third AAAI Conference on Arti_cial Intelligence (AAAI-19), Honolulu, HI, USA., February 2019.

[31] C. Veness, Calculate Distance and Bearing between Two Latitude/Longitude Points Using Haversine Formula in JavaScript, http://www.movable-type.co.uk/scripts/latlong. html, 2002-2020.

[32] M. A. Mohammed, H. A. Karrar, S. Alaa et al., "Benchmarking methodology for selection of optimal covid-19 diagnostic model based on entropy and topsis methods," IEEE Access, vol. 8, 2020.

[33] China Society for Industrial and Applied Mathematics, "Dataset: the data of allocated sharing tasks," 2017, http:// www.mcm.edu.cn/html_cn/node/460baf68ab0ed0e1e557a0c 79b1c4648.html. 